\title{
Comparative Docking Studies on Curcumin with COVID-19 Proteins
}

Renuka Suravajhala ${ }^{1 *}$, Abhinav Parashar ${ }^{2 *}$, Babita Malik ${ }^{1}$, Viswanathan Arun Nagaraj $^{3}$, Govindarajan Padmanaban $^{4}$, PB Kavi Kishor ${ }^{2}$, Rathnagiri Polavarapu ${ }^{5}$ and Prashanth Suravajhala ${ }^{6,7}$

${ }^{1}$ Department of Chemistry, Manipal University Jaipur, Jaipur 303 007, Rajasthan, India

${ }^{2}$ Department of Biotechnology, Vignan's Foundation for Science, Technology \& Research (Deemed to be

University), Vadlamudi, Guntur 522 213, Andhra Pradesh, India

${ }^{3}$ Institute of Life Sciences, Nalco Square, Chandrasekharpur, Bhubaneswar - 751023, Odisha

${ }^{4}$ Indian Institute of Science, Bengaluru 560 012, India

${ }^{5}$ Genomix CARL Pvt. Ltd., Pulivendula 516 390, Kadapa, Andhra Pradesh, India

${ }^{6}$ Department of Biotechnology and Bioinformatics, Birla Institute of Scientific Research, Jaipur 320001, Rajasthan, India

${ }^{7}$ Bioclues.org, India

*Equal contributing authors

Correspondence: giri@genomixbiotech.com and prash@bisr.res.in

\section{Highlights}

1. Our findings confirm the role of Q163 aminoacid site for potential tethered site or target which is in agreement with ivermectin, the best possible and known drug.

2. We have employed a rigorous strategy in screening the docking complexes from a majority of hypothetical genes or orphan open reading frames, structural and non-structural proteins.

3. We believe that the findings presented in our paper will appeal to researchers working on COVID-19, particularly those interested to characteristically screen docking complexes. 


\begin{abstract}
Corona virus disease 2019 (COVID-19) is caused by a Severe Acute Respiratory SyndromeCoronavirus 2 (SARS-CoV-2), which is a positive strand RNA virus. The SARS-CoV-2 genome and its association to SAR-CoV-1 vary from ca. $66 \%$ to $96 \%$ depending on the type of betacoronavirdeae family members. With several drugs, viz. chloroquine, hydroxychloroquine, ivermectin, artemisinin, remdesivir, azithromycin considered for clinical trials, there has been an inherent need to find distinctive antiviral mechanisms of these drugs. Curcumin, a natural bioactive molecule has been shown to have a therapeutic potential for various diseases, but its effect on COVID-19 has not been explored. In this study, we show the binding potential of curcumin targeted to a variety of SARS-CoV-2 proteins, viz. spike glycoproteins (PDB ID: 6VYB), nucleocapsid phosphoprotein (PDB ID: 6VYO), membrane glycoprotein (PDB ID: 6M17) along with nsp10 (PDB ID: 6W4H) and RNA dependent RNA polymerase (PDB ID: 6M71) structures. Our results indicate that curcumin has high binding affinity towards nucleocapsid and nsp 10 proteins with potential antiviral activity.
\end{abstract}

Keywords: Curcumin, COVID-19, nucleocapsid phosphoprotein, membrane glycoprotein, antiviral mechanism

\title{
Introduction
}

COVID-19 has caused unprecedented morbidity and mortality across the globe [1]. SARS-CoV-2 contains 29 proteins that includes 4 structural proteins - Spike, Envelope, Membrane and Nucleocapsid, and the rest nonstructural and adjunct proteins. A polyprotein is acted upon by an encoded protease giving rise to 16 of these proteins. Whereas the viral RNA genome is close to $30 \mathrm{~Kb}$ in size, the structural proteins are mainly involved in entry, replication, assembly and propagation of the virus into lung and other cells carrying the ACE2 receptor [2]

A large number of potential therapeutic molecules that include antibiotics, antivirals, and anti-malarials are being tested against COVID-19, that has caused global devastation. In a recent study, Sampangi-Ramiah et al., 2020, have evaluated 27 natural compounds for binding affinities to both the proteases of COVID-19 [3]. A 
few compounds have been found to have good binding affinities ranging from $-6.4 \mathrm{kcal} / \mathrm{mol}$ (for coriandrin) to $-8.0 \mathrm{kcal} / \mathrm{mol}$ (for glabridin) and $-8.1 \mathrm{kcal} / \mathrm{mol}$ (for glucobrassicin), comparable to the anti-HIV drug saquinavir. In the present study, we have evaluated the binding affinities of 14 drug candidates with SARSCov-2 proteins: spike glycoproteins (PDB ID: 6VYB), nucleocapsid phosphoprotein (PDB ID: 6VYO), membrane glycoprotein (PDB ID: 6M17) nsp10 (PDB ID: 6W4H) and RNA dependent RNA polymerase (PDB ID: 6M71) structures. We find that the natural molecule, curcumin from turmeric, has good binding affinities to nucleocapsid and nsp-10, comparable to those of ivermectin, azithromycin and remedesivir. Turmeric as a spice is used extensively in India and is described in ancient literature for its wide medicinal use even as numerous studies describing that food supplements and nutraceuticals may turn out to be long-term option to prevent the viral infection. For example, curcumin has been shown to have a strong anti-oxidative and anti-inflammatory properties and has potential therapeutic effects on chronic diseases [4], Noroviruses [5], Hepatitis B infection [6] and diabetes mediated cerebal infarction [7]. In addition to its profound immunomodulatory effects, it may also bring about changes by directly binding to crucial viral proteins and preventing their function. Hence, we attempt to understand the molecular interactions and decipher the role of curcumin with SARS-CoV-2 structural and non-structural proteins. To provide molecular details of ligand recognition and better understanding of the ligand binding behaviour of different targets/receptors, we determined characteristic features on ligand binding, safe dosage, $K_{i}$ values, and classify key factors that direct the docking complexes of drugs. The spike glycoproteins of SARS family to which SARS-CoV-2 and SARS$\mathrm{CoV}$ belong to, have structure resemblance with 8 amino acids related to 14 binding residues, and are known to be conserved in SARS-CoV-2 [8]. It was further shown that angiotensin converting enzyme-2 (ACE-2) interacts with the binding residues of SARS-CoV-2 $[9,10]$. We deliberate on the role of curcumin, its antiviral drug binding efficiency and calculate the dosage concentration using molecular modelling approaches targeted for therapeutics of SARS-CoV-2.

\section{Material and methods}

Preparation of ligands: The molecular docking studies were performed using AutoDock 4.2 software [11]. For the current study, 15 ligands were considered based on their current experimentation in treatment of 
COVID-19 patients, based on known potent antiviral/antimalarial properties and their plasma concentration (supplementary table 1). The ligands with 3D structures were retrieved from PubChem as sdf files, as others were subjected to a 3D structure generation on CORINA [12] using their SMILE nomenclature. Further, pdbqt files for the ligands were generated by OpenBabel [13].

Preparation of proteins and grid parameters: The protein data bank (PDB) structures of different COVID19 proteins were retrieved from RCSB membrane protein (6M17), polymerase (6M71), spike (6VYB), nucleocapsid (6VY0) and nsp10 (6W4H). The protein structures were visualized on PyMOL 1.3 or 2.5 [14] by demarcating the transmembrane regions if any, present in the protein. While the pdbqt files were generated for the proteins to initiate the grid parameters, we considered less than $1 \AA$ and $\mathrm{x}, \mathrm{y}, \mathrm{z}$ coordinates to establish the size of the protein for docking studies. MGL tool 1.5.6 was used for generating necessary protein and ligand files along with the respective grids and dock files and the grid generation and docking were subsequently executed through AutoGrid 4.2 and AutoDock 4.2 respectively [11]. To find the binding affinity of ligands with selected proteins, drug targets were screened from estimated free energy of binding and inhibition constant $\left(\mathrm{K}_{\mathrm{i}}\right)$ at $298.15 \mathrm{~K}$ temperature. The docked ligand-protein complexes were visualized on Chimera 1.12

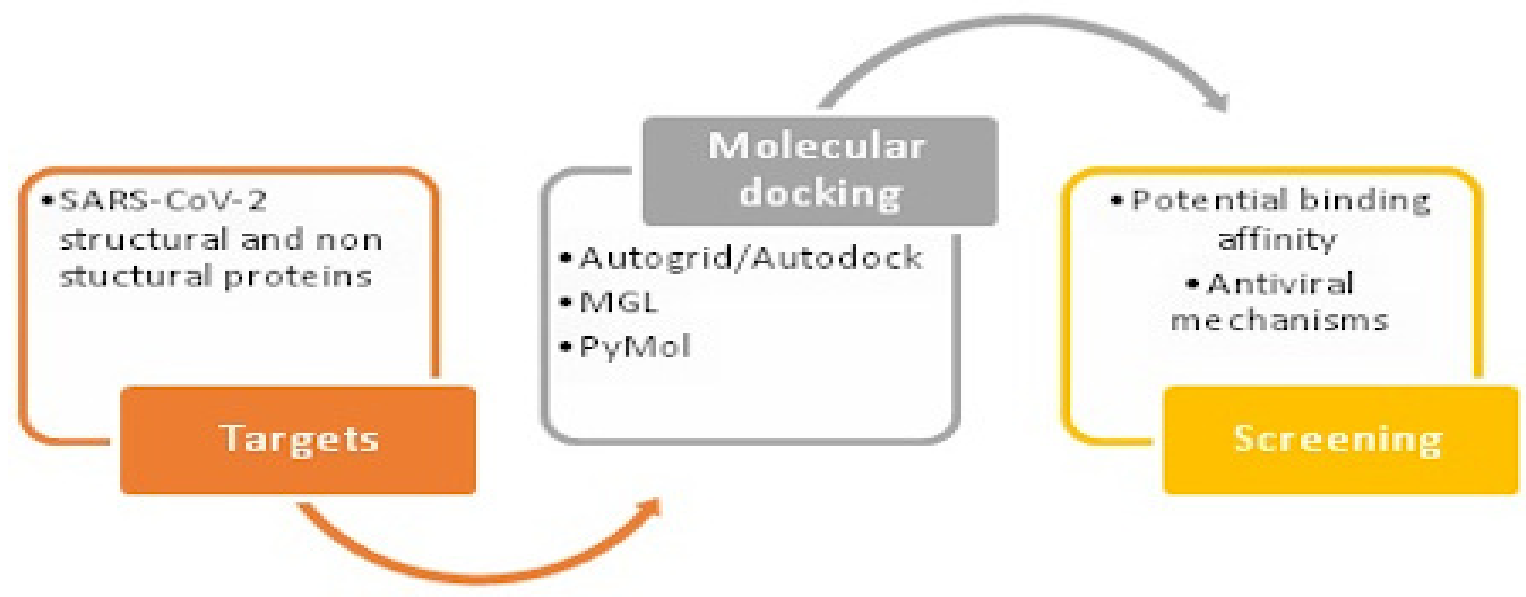

[15] for image construction. The binding energies and affinities for the ligands were obtained from the log files of the docks generated by AutoDock. A brief methodological overview is presented in Figure 1.

Figure 1: A pictorial methodology outlining molecular docking approaches 


\section{Results and Discussion}

Comprehensive docking studies were carried out on 14 drug molecules with antiviral properties that are considered for clinical trials along with curcumin using AutoDock 4.2. This resulted in free energy $(\Delta \mathrm{G})$ and inhibition constant $\left(\mathrm{K}_{\mathrm{i}}\right)$ values showing the binding interaction of the ligand molecules with different structural and non-structural proteins of SARS-CoV-2. From the docking studies, we observe that curcumin shows good binding affinity with nucleocapsid $\left(\Delta \mathrm{G}=-8.75 \mathrm{kcal} / \mathrm{mol}, K_{i}=0.39 \mu \mathrm{M}\right), \mathrm{nsp} 10\left(\Delta \mathrm{G}=-7.85 \mathrm{kcal} / \mathrm{mol}, K_{i}=1.77\right.$ $\mu \mathrm{M})$ which is comparable to quinidine with nucleocapsid $\left(\Delta \mathrm{G}=11.74 \mathrm{kcal} / \mathrm{mol}, K_{i}=2.50 \mathrm{nM}\right)$ and nsp10 $\left(\Delta \mathrm{G}=-8.45 \mathrm{kcal} / \mathrm{mol}, K_{i}=639 \mathrm{~nm}\right)$, ivermectin with nucleocapsid $\left(\Delta \mathrm{G}=-7.11 \mathrm{kcal} / \mathrm{mol}, \mathrm{K}_{\mathrm{i}}=6.17 \mu \mathrm{M}\right)$ and $\operatorname{nsp10}\left(\Delta \mathrm{G}=-9.82 \mathrm{kcal} / \mathrm{mol}, K_{i}=63.20 \mathrm{~nm}\right)$, azithromycin with nucleocapsid $\left(\Delta \mathrm{G}=-8.71 \mathrm{kcal} / \mathrm{mol}, K_{i}=0.41\right.$ $\mu \mathrm{M})$ and $\operatorname{nsp10}\left(\Delta \mathrm{G}=-8.03 \mathrm{kcal} / \mathrm{mol}, K_{i}=1.29 \mu \mathrm{M}\right)$ in nsp10), and remdesivir with nucleocapsid $(\Delta \mathrm{G}=$ $\left.-6.30 \mathrm{kcal} / \mathrm{mol}, K_{i}=23.94\right)$ and $\mathrm{nsp} 10\left(\Delta \mathrm{G}=-6.54 \mathrm{kcal} / \mathrm{mol}, K_{i}=16.02 \mu \mathrm{M}\right)($ Figure 2; Supplementary table-2). Ivermectin showed the best affinity towards all the targeted proteins studied and significant efficient binding to the non-structural proteins. The efficient binding to non-structural proteins is not much in the case of chloroquine, efavirenz, favipiravir and tenofovir, disoproxil.

Curcumin is a molecule with symmetrical halves containing a ring at both the edges (Supplementary figure 1). The bendable chain and the rigid rings of curcumin would serve a good clamp or a hook formation on the loop regions of targeting SARS-CoV-2 proteins. On the other hand, loops are known to play a major role in the stability of the protein structure [16] and hence a molecule binding to loop is a potential modulator of stability. Curcumin does not show a remarkably high $\mathrm{C}_{\text {-max }}$ value but the $K_{i}$ values correlate with binding to nucleocapsid and nsp10 proteins. We have visualised it using PYMOL and identified the best pose of curcumin with binding residues at L161, Q163, A173 of Loop 2 in nucleocapsid protein. We argue that the binding residues of loop 2, viz. Q163, L161, A173 in nucleocapsid protein may involve in a distinct signalling system associated with vertebrate evolution, as evident from the complex interaction pathways (Figure 2, panel). We have also identified K4281, L4365 of zinc finger groove in nsp10 protein as the binding residues. The zinc binding sites in nsp10 protein are HIT-type zinc finger family that are reported to bind to transcription regulatory proteins which help in recognizing RNA and also in protein-protein recognition [17]. Consequently, 
nsp10 plays a major role in viral transcription wherein nsp14 3'-5' exoribonuclease and nsp16 2'-Omethyltransferase are stimulated by playing a lead role in viral mRNAs cap methylation [18].
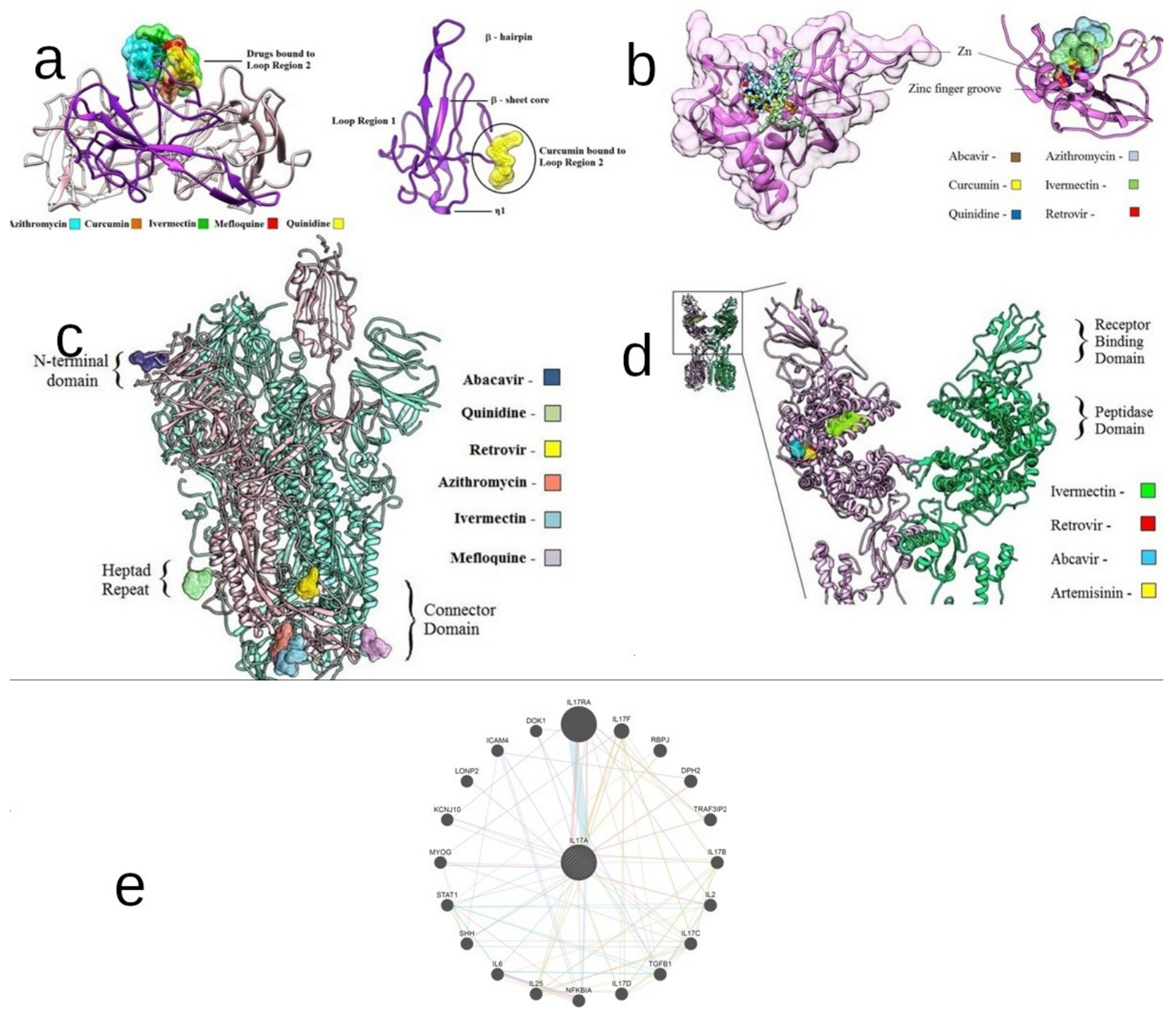

Figure 2: Protein-ligand interactions of (a) nucleocapsid phosphoprotein (PDB ID: 6VYO) (b) nsp10 (c) spike glycoproteins (PDB ID: 6VYB) and (d) membrane glycoprotein (PDB ID: 6M17). (e) Protein interaction map of interleukin-17, a human homologue of nucleocapsid which acts as a potent proinflammatory cytokine produced by activated memory $\mathrm{T}$ cells 
While nucleocapsid protein regulates replication and transcription of viral RNA, it also inhibits the EF1 $\alpha$ mediated protein translation, altering the cell cycle and further leads to apoptosis in host cells [19,20,21]. From our studies, we demonstrate that the drugs such as azithromycin, mefloquine, quinidine, ivermectin and curcumin show hydrogen binding interactions with Q163, A173, Q70, Q163, G164, L161, T165, L167 in loop 2 which serves as latent targets, activating T-cell cytotoxicity for candidate drug therapeutics. The crystal structure of the RNA binding domain of nucleocapsid protein of SARS-CoV-2 was recently published [22] which details the groove between the palm (loop region 2) and finger region (bsheet core dominantly) at the RNA binding. As loops are known to play a major role in the stability of a protein [16] they are flexible, and can relatively move with ease. We could, hence, predict the role of this loop in affecting the binding of RNA to the palm groove of nucleocapsid (Figure 2). Curcumin has shown exceptional binding affinity to this region, which therefore makes it a potent target drug for RNA binding domain or nucleocapsid of SARS-CoV-2. On the other hand, nsp16, which methylates the RNA cap with its S-adenosyl-L-methionine dependent methyl transferase activity, requires nsp10 as its stimulatory factor [23] as demonstrated in SARS-CoV, and the same is expected to be established in SARSCoV-2. At the same time, inhibiting nsp10 would not allow the viral RNA to camouflage with the eukaryotic RNA of humans and eventually inhibit the replication of virus in the host cell. Among six other drug molecules, we observed that curcumin also shows a very strong affinity to nsp10 and less than $2 \mathrm{mM}$ of the same is adequate concentration correlated with its $K_{i}$ constant. The binding of all these drugs is observed on the loop in the groove between the zinc fingers. Such strong affinity indicates that it is a probable target site for COVID-19. Thus, curcumin acts as a potential inhibitor of the nucleocapsid (6VYO), which is a multifunctional pro-inflammatory protein [19]. The regions are also exposed further considering them as epitope targets and is in agreement with the reports that curcumin, nanocurcumin inhibits transcription factor NFkB thereby leading to instability of pro-inflammatory cytokines such as interleukins and TNF-a [24]. Furthermore, curcumin is reported to suppress components of cellular signalling transduction pathways that play a key role in infected cells growth, and transformation by inhibiting protein kinases, activating enzyme cyclooxygenase (COX)-2 [25]. Studies related to spike proteins through their $\mathrm{S}^{\mathrm{B}}$ domain (at the N-terminus) interact with ACE2 to make an entry in the host cell [8]. This makes spike proteins, and more precisely the $\mathrm{S}^{\mathrm{B}}$ domain, a hot spot for therapy. Other two important regions of spike proteins are heptad 
repeats (HR1 and HR2), involved in viral fusion [26], and connector domain, which connects the two heptad repeats and it helps in stabilizing the post fusion structure [27]. We obtained six ligands keeping less than 50 $\mu \mathrm{M} \mathrm{K} \mathrm{K}_{\mathrm{i}}$ value as the criteria. Curcumin although shares site with mefloquine, is not a good inhibitor of the Spike protein, but binds to the loop of the connector domain of spike protein with a fair binding energy of -4.9 $\mathrm{kcal} / \mathrm{mol}$ corresponding to $\sim 250 \mathrm{mM} K_{i}$ value. Curcumin falls in the top 3 hydrophobic molecules with a $\operatorname{logS}$ value making it one of the best drug candidate molecules (Supplementary table 4). The spike proteins and membrane proteins readily come in contact with the cytoplasm of the host cell and therefore would have to come across a higher concentration of the drugs administered to the patient as compared to the proteins inside the coat (nucleocapsid, nsp10 and RDRP) given that the drug has to cross the surface protein and lipid layer of the virus. Taking into account of this fact, we observed that most of the drugs do not have a $\mathrm{C}$-max-- value of more than $5 \mathrm{mg} / \mathrm{mL}$, even the smallest compound with a molecular weight of $\sim 100 \mathrm{~g} / \mathrm{mol}$, e.g. favipiravir ( $\sim 150 \mathrm{~g} / \mathrm{mol}$ ), would be found at a concentration of $50 \mathrm{mM}$ ( the actual $\mathrm{C}_{\mathrm{mzx}}$ of favipiravir is $\sim 40 \mathrm{mg} / \mathrm{mL}$ ). Of that, if $5 \%$ of the drug manages to reach inside the viral coat, it would account to $2.5 \mu \mathrm{M}$ of the drugs available to act on nucleocapsid, nsp10 and/or RDRP, hence making the cut-off value for core proteins.

Ivermectin and azithromycin both have a complex structure with multiple rings and they bind at the same locus with similar affinities at the C-terminal of the spike protein. These drugs show interaction with the connector domain, indicating their stability and transition from pre-fusion to post fusion state rather than a preferable ACE2 receptor blocker. The in silico docking studies revealed considerable affinity towards a few drugs, viz. abcavir, artemisinin, ivermectin and retrovir at the peptidase domain of ACE2 segment of the complex. Whereas the viral domain $\left(\mathrm{B}^{0} \mathrm{AT} 1\right)$ showed considerable affinity only towards ivermectin $\left(\Delta \mathrm{G}=-7.66, \mathrm{~K}_{\mathrm{i}}=\right.$ $2.44 \mathrm{mM}$ ), yet that was not at the contact site of ACE2 and $\mathrm{B}^{0} \mathrm{AT} 1$. We further argue that ivermectin is relatively a complex molecule with reasonably high number of rings, with least $\mathrm{C}_{\max }$ and relatively most insoluble compound among the ones used here. The low $K_{i}$ values observed for ivermectin correlates with its low physiological $\mathrm{C}_{\max }$, which therefore could make a viable target drug. Curcumin, although has a binding site on both the human domain and the viral domain of the complex, it would need 10 times more concentration to act on them, given its $\mathrm{C}_{\max }$ value. A binding at the linker region would have been of great value for the 
therapeutics purpose, which was not observed for the given molecules. In addition, binding of these drugs even at the peptidase domain of ACE2 would inhibit the rotation of the same and hence the change between open and closed conformation, which facilitates the viral attachment [28]. Receptor binding domain (RBD), above the peptidase domain of ACE2, is identified only in the closed state, probably indicating the role of closed state in anchoring, hence the necessity of transition between the conformations for the invasion.

\section{Conclusions}

We propose curcumin as a therapeutic target for anti-corona virus drug development. In this work, we screened 14 ligands against SARS-CoV-2 structural and non-structural proteins and evaluate the binding affinity of curcumin to that of other known drugs that are commercially available. From the current study, it is evident that curcumin could play a major role in regulating the activity of nucleocapsid and nsp10, both of which are related to the detection and processing of viral RNA. At very high concentrations, curcumin acts on the spike and membrane proteins and hence effect on invasion itself is difficult to achieve with the given solubility and plasma concentrations of curcumin. Further study on a combinatorial administration of drugs with curcumin could demonstrate synergistic effects. Despite extensive studies and clinical trials for a variety of diseases, mostly cancers, there has been a view that curcumin is not an ideal drug candidate in view of its low bioavailability and stability in plasma. However, curcumin has been shown to be an effective adjunct drug or a food supplement with many strategies reported to enhance its bioavailability [28]. Nevertheless, even with low bioavailability, it is a very effective immunomodulator to cure experimental malaria [29], cerebral malaria [30] and infectious diseases [31]. The present study shows that in addition to being a potent immunomodulator, it can effectively bind and inhibit the nucleocapsid and nsp 10 proteins of COVID-19. Curcumin can be an ideal adjunct drug, being a food additive and non-toxic to humans at a dosage as high as 8 g/day [32]. 
Author contributions: GP, PBK and RP ideated the project. RS and AP jointly analysed the structures and modelled the docking complexes. PS performeddid the protein interaction analyses. AP and RS wrote the first draft with PS, PBK, BM and RP. GP, PBK, PS, VAN and RP proofread the manuscript.

Acknowledgements: Renuka Suravajhala gratefully acknowledges Manipal University Jaipur towards Ramdas Pai fellowship rendered to her.

Funding: None.

Competing interests: None

\section{References}

[1] www.who.int. last accessed: May 30, 2020.

[2] Chen, Y., Liu, Q., Guo, D. Emerging coronaviruses: Genome structure, replication, and pathogenesis. Journal of Medical Virology 92,(2020) 418-423 . https://doi.org/10.1002/jmv.25681.

[3] Sampangi-Ramaiah, M.H., Vishwakarma, R. and Shaanker, R.U., Molecular docking analysis of selected natural products from plants for inhibition of SARS-CoV-2 main protease. Current Science , 118(7), 10871092 (2020).

[4] Padmanaban, G., Nagaraj, V. A. Curcumin from turmeric as an adjunct drug? Chem. 57,(2018) 179-202. https://doi.org/10.1016/B978-0-444-64057-4.00006-5.

[5] Yang, M., Lee, G., Si, J., Lee, S. J., You, H. J., Ko, G. Curcumin shows antiviral properties against norovirus. Molecules, 21(10),(2016) 1401. https://doi.org/10.3390/molecules21101401. 
[6] Hesari, A., Ghasemi, F., Salarinia, R., Biglari, H., Tabar Molla Hassan, A., Abdoli, V., Mirzaei, H. Effects of curcumin on NF- $\mathrm{B}, \mathrm{AP}-1$, and $\mathrm{Wnt} / \beta$-catenin signaling pathway in hepatitis B virus infection. Journal of cellular biochemistry, 119(10), (2018) 7898-7904. https://doi.org/10.1002/jcb.26829.

[7] Xia, M., Ye, Z., Shi, Y., Zhou, L., \& Hua, Y. Curcumin improves diabetes mellitus-associated cerebral infarction by increasing the expression of GLUT1 and GLUT3. Molecular medicine reports, 17(1), (2018) 1963-1969. https://doi.org/10.3892/mmr.2017.8085.

[8] Walls, A. C., Park, Y. J., Tortorici, M. A., Wall, A., McGuire, A. T., Veesler, D. Structure, function, and antigenicity of the SARS-CoV-2 spike glycoprotein. Cell. (2020) 281-292.e6. https://doi.org/10.1016/j. cell .2020.02.058.

[9] Hoffmann M., Kleine-Weber H., Schroeder S., Krüger N., Herrler T., Erichsen S. SARS-CoV-2 cell entry depends on ACE2 and TMPRSS2 and is blocked by a clinically proven protease inhibitor. Cell. 2020;S00928674(20):30229-30234. doi: 10.1016/j.cell.2020.02.052.

[10] Rossi GP, Sanga V, Barton M. Potential harmful effects of discontinuing ACE-inhibitors and ARBs in COVID-19 patients. Elife. 2020;9:e57278. Published 2020 Apr 6. doi:10.7554/eLife.57278

[11]Morris, G. M. et al. AutoDock4 and AutoDockTools4: automated docking with selective receptor flexibility. J. Comput. Chem.30, (2009) 2785-2791 . https://doi.org/10.1002/jcc.21256.

[12]_CORINA/MN-AM website: https://www.mn-am.com/ Last accessed: June 5, 2020.

[13] N M O'Boyle, M Banck, C A James, C Morley, T Vandermeersch, and G R Hutchison. "Open Babel: An open chemical toolbox." J. Cheminf. (2011), 3, 33. DOI:10.1186/1758-2946-3-33

[14] DeLano, W. L. (2009). The PyMOL Molecular Graphics System; DeLano Scientific: San Carlos, CA, 2002. There is no corresponding record for this reference. 
[15]Pettersen, E.F., Goddard, T.D., Huang, C.C., Couch, G.S., Greenblatt, D.M., Meng, E.C., and Ferrin, T.E. "UCSF Chimera - A Visualization System for Exploratory Research and Analysis." J. Comput. Chem. 25(13):1605-1612 (2004).

[16] Balasco, N., Esposito, L., De Simone, A. D., Vitagliano, L. Role of loops connecting secondary structure elements in the stabilization of proteins isolated from thermophilic organisms. Protein Sci. 22, (2013) 10161023. https://doi.org/10.1002/pro.2279.

[17]Su, D., Lou, Z., Sun, F., Zhai, Y., Yang, H., Zhang, R., Joachimiak, A., Zhang, X. C., Bartlam, M., Rao, Z. Dodecamer structure of severe acute respiratory syndrome coronavirus nonstructural protein nsp10. Journal of Virology, 80(16), (2006) 7902-7908. https://doi.org/10.1128/JVI.00483-06.

[18] Bouvet M, Lugari A, Posthuma CC, et al. Coronavirus Nsp10, a critical co-factor for activation of multiple replicative enzymes. J Biol Chem. 2014;289(37):25783-25796. doi:10.1074/jbc.M114.577353.

[19] McBride, R., van Zyl, M., Fielding, B. C. The coronavirus nucleocapsid is a multifunctional protein. Viruses, 6(8),(2014) 2991-3018. https://doi.org/10.3390/v6082991.

[20] Hilgenfeld R. From SARS to MERS: crystallographic studies on coronaviral proteases enable antiviral drug design. The FEBS journal, 281(18), 4085-4096(2014). https://doi.org/10.1111/febs.12936Kang, S. et al. Crystal structure of SARS-CoV-2 nucleocapsid protein RNA binding domain reveals potential unique drug targeting sites. Acta Pharm. Sin. B (2020 Apr 20). https://doi.org/10.1016/j.apsb.2020.04.009, Pubmed:32363136.

[21] Zhou, B., Liu, J., Wang, Q., Liu, X., Li, X., Li, P., Ma, Q., Cao, C. The nucleocapsid protein of severe acute respiratory syndrome coronavirus inhibits cell cytokinesis and proliferation by interacting with translation elongation factor 1alpha. Journal of Virology, 82(14), (2008) 6962-6971. https://doi.org/10.1128/JVI.00133$\underline{08}$ 
[22] Kang S, Yang M, Hong Z, et al. Crystal structure of SARS-CoV-2 nucleocapsid protein RNA binding domain reveals potential unique drug targeting sites [published online ahead of print, 2020 Apr 20]. Acta Pharm Sin B. 2020;10.1016/j.apsb.2020.04.009. doi:10.1016/j.apsb.2020.04.009

[23] Chen, Y. et al. Biochemical and structural insights into the mechanisms of SARS coronavirus RNA ribose 2'-O-methylation by nsp16/nsp10 protein complex. PLOS Pathog. 7, (2011)e1002294. https://doi.org/10.1371/journal.ppat.1002294.

[24]Dolati S, Ahmadi M, Aghebti-Maleki L, et al. Nanocurcumin is a potential novel therapy for multiple sclerosis by influencing inflammatory mediators. Pharmacol Rep. 2018;70(6):1158-1167. doi:10.1016/j.pharep.2018.05.008

[25] Prasad, S., Tyagi, A. K., Aggarwal, B. B. Recent developments in delivery, bioavailability, absorption and metabolism of curcumin: the golden pigment from golden spice. Cancer Res. Treat. Off. J. Korean Cancer Assoc. 46, (2014) 2-18. https://doi.org/10.4143/crt.2014.46.1.2.

[26]Bosch, B. J., van der Zee, R., de Haan, C. A., Rottier, P. J. The coronavirus spike protein is a class I virus fusion protein: structural and functional characterization of the fusion core complex. J. Virol. 77, (2003 ) 88018811. https://doi.org/10.1128/jvi.77.16.8801-8811.2003.

[27] Walls, A. C., Tortorici, M. A., Snijder, J., Xiong, X., Bosch, B. J., Rey, F. A., Veesler, D. Tectonic conformational changes of a coronavirus spike glycoprotein promote membrane fusion. Proceedings of the National Academy of Sciences, 114(42),114, (2017) 11157-11162. https://doi.org/10.1073/pnas. 1708727114.

[28]Nelson, K. M., Dahlin, J. L., Bisson, J., Graham, J., Pauli, G. F., \& Walters, M. A. The essential medicinal chemistry of curcumin: miniperspective. Journal of medicinal chemistry, 60(5),(2017) 1620-1637. https://doi.org/10.1021/acs.jmedchem.6b00975. 
[29] Vathsala PG, Dende C, Nagaraj VA, Bhattacharya D, Das G, Rangarajan PN, Padmanaban G. Curcuminarteether combination therapy of Plasmodium berghei-infected mice prevents recrudescence through immunomodulation. PLoS One. 7(1), (2012) e29442. https://doi.org/10.1371/journal.pone.0029442.

[30] Dende, C., Meena, J., Nagarajan, P., Nagaraj, V. A., Panda, A. K., \& Padmanaban, G. Nanocurcumin is superior to native curcumin in preventing degenerative changes in Experimental Cerebral Malaria. Scientific reports, 7(1), (2017) 1-12. https://doi.org/10.1038/s41598-017-10672-9.

[31] Padmanaban, G., Nagaraj, V. A. Curcumin may defy medicinal chemists. ACS Med. Chem. Lett. 8, (2017) 274 . https://doi.org/10.1021/acsmedchemlett.7b00051.

[32]Soleimani, V., Sahebkar, A., Hosseinzadeh, H. Turmeric (Curcuma longa) and its major constituent (Curcumin) as nontoxic and safe substances: Review. Phytother. Res. 32,(2018) 985-995. https://doi.org/10.1002/ptr.6054. 